\title{
Investigator Status
}

National Cancer Institute

\section{Source}

National Cancer Institute. Investigator Status. NCI Thesaurus. Code C70723.

A category used to describe the registration status of a clinical study investigator with the regulatory authority agency, such as the FDA, or the investig ator activity status within a particular study. 